\title{
Investigation of a family of cubic dynamic systems
}

\author{
Alexey Andreev ${ }^{1}$, Irina Andreeva ${ }^{2}$ \\ ${ }^{1}$ St. Petersburg State University, St. Petersburg, Russia \\ ${ }^{2}$ Peter the Great St. Petersburg Polytechnic University, St. Petersburg, Russia \\ ${ }^{2}$ Corresponding author \\ E-mail: ${ }^{1}$ irandr@inbox.ru, ${ }^{2}$ irandr.andreeva@yandex.ru
}

Received 1 November 2017; accepted 12 November 2017

DOI https://doi.org/10.21595/vp.2017.19389

Check for updates

Abstract. A family of dynamic systems acting on a real plane $x, y$ has been considered, which polynomial right parts are reciprocal forms of $x$ and $y$, one is a cubic, and another is a square form. A problem to reveal all topologically different phase portraits possible for these systems in a Poincare circle with coefficient criteria of every portrait's realization has been solved. A Poincare method of serial mappings - central and orthogonal - has been applied. Qualitative and quantitative results for phase portraits have been given. All stages of a solution process are described.

Keywords: dynamic system, limit cycles, phase portraits, singular points, separatrices, trajectories.

\section{Introduction}

A modern theory of dynamic systems appears to be a synthetic field of investigations including methods of different areas of mathematics such as topology and algebra, differential equations and algebraic geometry. The results are used in the fields of a dynamic chaos theory, synergetics, nonequilibrium thermodynamics etc.

According to Jules H. Poincare, a normal autonomous second-order differential system with polynomial right parts in principle allows its full qualitative investigation on an extended arithmetical plane $\bar{R}_{x, y}^{2}[1]$. Since that time investigations in this field have been conducted for a series of such systems' types, e.g. for quadratic systems [2], for homogeneous cubic systems, for systems containing nonzero linear terms and for systems with homogeneous nonlinear terms of the third [3], the fifth and seventh degrees [4] in their right parts, for which a singular point $\mathrm{O}(0,0)$ appears to be a center or a focus, and for some other types of dynamic systems.

Let us consider a broad family of dynamical systems acting on an arithmetical plane $x, y$ :

$\frac{d x}{d t}=X(x, y), \frac{d y}{d t}=Y(x, y)$.

Such as $X(x, y), Y(x, y)$ are reciprocal forms of $x$ and $y, X$ be a cubic, and $Y$ be a square form, such as $X(0,1)>0, Y(0,1)>0$. A problem is formulated to reveal all topologically different types of phase portraits possible for Eq. (1) systems in a Poincare circle and outline close to coefficient criteria of each portrait's appearance. In order to solve this problem we use a method of serial mappings of Jules Henri Poincare: 1$)$ a central (i.e. from a center $(0,0,1)$ of a sphere $\sum: X^{2}+Y^{2}+Z^{2}=1$ ) mapping of a plane $x, y$, augmented with a line at infinity (i.e. $\bar{R}_{x, y}^{2}$ plane) on a sphere $\sum$ with identified diametrically opposite points, 2) an orthogonal mapping of a lower enclosed semi sphere of a sphere $\sum$ to a circle $\bar{\Omega}: x^{2}+y^{2} \leq 1$ with identified diametrically opposite points of its boundary $\Gamma$. The sphere $\sum$ and the circle $\bar{\Omega}$ are called in this process the Poincare sphere and the Poincare circle correspondingly [1].

\section{Part 1}

In the Part 1 of this work we present a solution of an assigned problem for those Eq. (1) systems, which decompositions of forms $X(x, y), Y(x, y)$ into a real forms of lower degrees 
contain 3 and 2 multipliers correspondingly:

$X(x, y)=p_{3}\left(y-u_{1} x\right)\left(y-u_{2} x\right)\left(y-u_{3} x\right), \quad Y(x, y)=c\left(y-q_{1} x\right)\left(y-q_{2} x\right)$,

where $p_{3}>0, c>0, u_{1}<u_{2}<u_{3}, q_{1}<q_{2}, u_{i} \neq q_{j}$ for each $i$ and $j$.

The solution process contains the follows steps.

Basic Concepts and Notations.

The following notations are introduced for an arbitrary system under consideration in the Part 1.

$P(u), Q(u)$ - it's polynomials $P, Q$ :

$P(u):=X(1, u) \equiv p_{3}\left(u-u_{1}\right)\left(u-u_{2}\right)\left(u-u_{3}\right)$,

$Q(u):=Y(1, u) \equiv c\left(u-q_{1}\right)\left(u-q_{2}\right)$.

$R S P(R S Q)$ - an ascending sequence of all real roots of this system's polynomial $P(u)$ $(Q(u)), R S P Q$ - an ascending sequence of all real roots of both this system's polynomials $P(u)$, $Q(u)$.

The DC-transformation is a double change of variables in this system: $(t, y) \rightarrow(-t,-y)$. It transforms it into another such system, signs and numberings of roots of polynomials $P(u), Q(u)$ and direction of trajectories' motion corresponding to increasing $t$ are reversed. Two different Eq. (2) systems are called mutually inversed (relatively to a DC-transformation), if a DC-transformation converts one of them into another, and independent (of a DC-transformation) in an opposite case.

Evidently, for an arbitrary Eq. (2) system are possible 10 different types of $R S P Q$, because $C_{5}^{2}=5 ! / 3 ! 2 !=10$.

The DC-transformation of Eq. (2) systems shows, that six of them are independent in pairs, while each system among the rest has the mutually inversed one among the first six systems. To each one of different $R S P Q^{\prime} S$ of the Eq. (2) system we assign the definite number $r \in\{1, \ldots, 10\}$ such as $R S P Q$ with numbers $r=\overline{1,6}$ appear to be independent in pairs, and sequences with numbers $r=\overline{7,10}$ mutually inversed to those with numbers $r=\overline{1,4}$ correspondingly.

The notion of a family number $r$ of Eq. (2) systems is introduced:

A family number $r$ of Eq. (2) systems := a totality of all systems of Eq. (2) family, for every of them $R S P Q$ has the number $r$.

Further according to a common scheme we alternately study families of Eq. (1) systems with numbers $r=\overline{1,6}$. The results relating to families, $r=\overline{7,10}$, we obtain from the results previously found for families, $r=\overline{1,4}$, using the DC-transformation of them.

The process of a study of a fixed Eq. (2) family contains the following steps.

I) Here we enlist singular points of systems belong to the chosen family in a Poincare circle $\bar{\Omega}$ : they are a point $O(0,0) \in \Omega$ and points $O_{i}^{ \pm}\left(u_{i}, 0\right) \in \Gamma, i=\overline{0,3}, u_{0}=0$. For each singular point we introduce notions of bundles $\mathrm{N}$ (node) and $\mathrm{S}$ (saddle) of semi trajectories of systems of the family, adjacent to this singular point; of the topodynamical type of the singular point (TD - type), and a separatrix of the singular point.

II) A family is further broken into subfamilies having the numbers $s=\overline{1,7}$. For each subfamily we find TD - types of all singular points of the systems and their separatrices.

III) $\forall s \in\{1, \ldots, 7\}$ we study the behavior of separatrices of singular points of systems belonging to the subfamily and answer the questions about a uniqueness of continuation of each separatrix from a small neighborhood of a singular point to all its lengths and about a mutual arrangement of all separatrices in a Poincare circle $\Omega$.

IV) We construct phase portraits of systems belonging to a chosen family in the both forms such as a graphical form and a table form. The criteria of a realization of each phase portrait are indicated. 
The result of the Part 1 of this work is the follows: for the systems belonging to the family number $r=1$ there are 25 different phase portraits possible, for the systems of families number 2 and number 3-9 phase portraits per each family, for families with numbers 4 and 5-7 per each family, for systems of the 6th family - 36, and this means that for all types of Eq. (2) systems appear to exist 93 different types of phase portraits. It's a considerable amount. But we must remember that each family contains an uncountable number of special dynamic systems.

\section{Part 2}

In the following parts 2 and 3 the problem has been solved for a Eq. (3) family. The Eq. (3) family of Eq. (1) systems is introduced, i.e. a totality of all Eq. (1) systems, for each of them decompositions of forms $X(x, y), Y(x, y)$ into a real multipliers of lowest degrees contain two multipliers each:

$X(x, y)=p\left(y-u_{1} x\right)^{k_{1}}\left(y-u_{2} x\right)^{k_{2}}, \quad Y(x, y)=q\left(y-q_{1} x\right)\left(y-q_{2} x\right)$,

where $p, q, u_{1}, u_{2} q_{1}, q_{2} \in R, p>0, q>0, u_{1}<u_{2}, q_{1}<q_{2}, u_{i} \neq q_{j}$ for each $i, j \in\{1,2\}$, $k_{1}, k_{2} \in N, k_{1}+k_{2}=3$.

It's naturally to distinguish two classes of Eq. (3) systems. The A class contains systems with $k_{1}=1, k_{2}=2$, and the $\mathrm{B}$ class contains systems with $k_{1}=2, k_{2}=1$.

In the Part 2 of this work we give a full solution of the assigned task for the systems belonging to the A class of the Eq. (3) family, i.e.:

$\frac{d x}{d t}=p\left(y-u_{1} x\right)\left(y-u_{2} x\right)^{2}, \frac{d y}{d t}=q\left(y-q_{1} x\right)\left(y-q_{2} x\right)$.

The process of the solution contains steps similar to the ones described in a previous Part 1 of this article.

For an arbitrary Eq. (4) system we introduce the following concepts.

$P(u), Q(u)$ - it's polynomials $P, Q$ :

$P(u):=X(1, u) \equiv p\left(u-u_{1}\right)\left(u-u_{2}\right)^{2}, Q(u):=Y(1, u) \equiv q\left(u-q_{1}\right)\left(u-q_{2}\right)$.

$R S P(R S Q)$ - is an ascending sequence of all real roots of the system's polynomial $P(u)$ $(Q(u)), R S P Q$ - is an ascending sequence of all real roots of both system's polynomials $P(u)$ and $Q(u)$. There exist 6 different possible variants of $R S P Q$ due to $C_{4}^{2}=4 ! / 2 ! 2 !=6$. These variants are numbered in some order from 1 to 6 .

Further we introduce a notion of a family number $r$ of Eq. (4) systems:

A family number $r$ of Eq. (4) systems is a totality of all Eq. (4) systems which have the same $R S P Q$ number $r$ from the above mentioned list of possible variants.

Alternate study of families of Eq. (4) systems.

A process of study of each fixed family includes the follows steps.

I) For each and every singular point of an arbitrary system of this family we introduce the notions of the bundles $\mathrm{N}$ (node) and S (saddle) of semi trajectories of the system, adjacent to this singular point; of its separatrix; of its topodynamical type (TD-type).

II) A family is broken into subfamilies with numbers $s=\overline{1,5 .} \forall s \in\{1, \ldots, 5\}$ we find TDtypes of singular points of systems belonging to subfamilies, and their separatrices.

III) $\forall s \in\{1, \ldots, 5\}$ we study the behavior of separatrices of singular points of systems belonging to a chosen subfamily and answer the questions about a uniqueness of global continuation of each separatrix from a small neighborhood of a singular point to all its lengths in the circle $\Omega$, and also about a mutual arrangement of all separatrices in a Poincare circle $\Omega$. If for a fixed $s$ the global continuation of each separatrix of singular points of the subfamily of systems 
is unique, their mutual arrangement in a Poincare circle $\Omega$ is invariable, and consequently all systems of a chosen subfamily have in a Poincare circle one common topological type of a phase portrait. Oppositely, if for some number $s$ of a subfamily appears to be that systems belonging to this subfamily have several, for instance $m$ separatrices which global continuations are not unique, then this subfamily is broken into $m$ additional subfamilies of the next order, for every of which, as we revealed in their further study, the global continuation of each separatrix is unique, their mutual arrangement in the circle $\Omega$ is invariable, and as a result the topological type of a phase portrait of all this family of systems in the $\bar{\Omega}$ circle is common for the family under consideration.

IV) We construct phase portraits in the circle $\bar{\Omega}$ for the systems of Eq. (4) families, $r=\overline{1,6}$, in the graphical and the table forms and indicate criteria of their realization for each portrait.

The result of the Part 2 is the follows: Eq. (4) systems of the family number lhave in the $\bar{\Omega}$ circle 13 different phase portraits, Eq. (4) systems of the family number $2-7$, number $3-10$, number 4, 5 and 6-5 per each type, and totally all Eq. (3) systems of the A class show 45 different phase portraits.

\section{Part 3}

In the Part 3 the full solution of our task for the Eq. (3) systems of the B class is given:

$$
\frac{d x}{d t}=p\left(y-u_{1} x\right)^{2}\left(y-u_{2} x\right), \frac{d y}{d t}=q\left(y-q_{1} x\right)\left(y-q_{2} x\right) .
$$

For an arbitrary Eq. (5) system $P(u), Q(u)$ - it's polynomials $P, Q$ :

$P(u):=X(1, u) \equiv p\left(u-u_{1}\right)^{2}\left(u-u_{2}\right), \quad Q(u):=Y(1, u) \equiv q\left(u-q_{1}\right)\left(u-q_{2}\right)$.

$R S P Q$ shows us 6 different variants, because $C_{4}^{2}=4 ! / 2 ! 2 !=6$.

The result of the investigation in the Part 3 is the follows: all the Eq. (5) family of dynamic systems is broken into 52 different subfamilies, and all systems of every subfamily have in a Poincare circle $\bar{\Omega}$ one common, belonged to this special subfamily, topological type of a phase portrait. As a result, we have constructed 52 different phase portraits in this case.

\section{Part 4}

In the Part 4 we solve the problem for a Eq. (6) family, i.e. for a family of Eq. (1) systems:

$$
\begin{aligned}
& \frac{d x}{d t}=p_{3}\left(y-u_{1} x\right)\left(y-u_{2} x\right)\left(y-u_{3} x\right), \quad \frac{d y}{d t}=c\left(y-q_{1} x\right)^{2}, \\
& p_{3}>0, \quad c>0, \quad u_{1}<u_{2}<u_{3}, \quad q(\in R) \neq u_{i}, \quad i=\overline{1,3} .
\end{aligned}
$$

The solution process includes the follows steps. Let's brake the Eq. (6) family into subfamilies numbered with $r=\overline{1,4}$.

Each of those is a totality of systems with an $R S P Q$ number $r$, where $r$ is its number in the list of possible $R S P Q s$ :

1. $u_{1}, u_{2}, u_{3}, q$,

2. $u_{1}, u_{2}, q, u_{3}$,

3. $u_{1}, q, u_{2}, u_{3}$,

4. $q, u_{1}, u_{2}, u_{3}$.

Applying to the Eq. (6) system a double change of variables (DC): $(t, y) \rightarrow(-t,-y)$, we reveal, that it transforms families of these systems having the numbers $r=1,2,3,4$, into their families with numbers $r=4,3,2,1$ correspondingly, and backwards. We note: this fact means, that families of Eq. (6) systems having numbers 1 and 2 are not connected with the DC- 
transformation; the same time families number 3 and 4 are mutually inversed relatively the DCtransformation to the families number 2 and 1 correspondingly.

I) We study alternately families of systems, $r=1,2$, following the common program of Eq. (1) systems study [5], i.e.:

1. We fix $r \in\{1,2\}$, then we brake the chosen family into subfamilies numbered with $s[5,6]$, $s=\overline{1,9}$, and find the topodynamical types (TD-types) of singular points of these systems.

2. $\forall s \in\{1, \ldots, 9\}$ we construct an "Off-Road Map" (ORM) for the systems of a chosen subfamily [5-7], and find using it $\alpha(\omega)$ - limit set of each $\alpha(\omega)$ - separatrix of these systems, and reveal the mutual arrangement of all separatrices in a Poincare circle.

3 . We construct all different phase portraits for systems under consideration.

II) We study alternately families of systems, $r=3,4$, using the DC-transformation of the results obtained for families, $r=2,1$. Then we construct all different phase portraits for the families of systems number 3 and 4 .

III) Summarize the results of our studies. For the Eq. (6) systems of families with numbers 1 , 2,3 and 4 there are $15+11+11+15=52$ different phase portraits in a Poincare circle $\bar{\Omega}$ possible.

\section{Part 5}

In the Part 5 we give the full solution of the problem for the Eq. (7) systems, i.e. for the Eq. (1) systems of the kind:

$\dot{x}=p_{0} x^{3}+p_{1} x^{2} y+p_{2} x y^{2}+p_{3} y^{3} \equiv p_{3}\left(y-u_{1} x\right)^{2}\left(y-u_{2} x\right)$,

$\dot{y}=x^{2}+b x y+c y^{2} \equiv c(y-q x)^{2}$,

where $p_{3}>0, c>0, u_{1}<u_{2}, q(\in R) \neq u_{1,2}$.

The process of study of these systems is quite similar to previously described for other families of Eq. (1) systems. For an arbitrary Eq. (7) system $P(u), Q(u)$-it's polynomials $P, Q$ :

$P(u):=X(1, u) \equiv p_{3}\left(u-u_{1}\right)^{2}\left(u-u_{2}\right), \quad Q(u):=Y(1, u) \equiv c(u-q)^{2}$,

and its $R S P Q$ shows 3 different variants.

The result of our study for this kind of systems is the follows. We proved, that for each family of Eq. (7) systems 7 topologically different phase portraits are possible, so for all three families of them, $r=\overline{1,3}$, the number of topologically different phase portraits equals to 21 .

\section{Conclusions}

We have constructed all above mentioned phase portraits both ways in a graphical and in a table (descriptive) forms. Each portrait has been described in a table, containing from 5 to 6 lines. Every single line describes in detail one invariant cell of the phase portrait: its boundary, a source and a sink of its phase flow. Such a table is called a descriptive phase portrait [8].

\section{References}

[1] Andronov A. A., Leontovich E. A., Gordon I. I., Mayer A. G. Qualitative Theory of Second-Order Dynamic Systems. John Wiley and Sons, New York, 1973.

[2] Andreev A. F., Andreeva I. A. On limit and separatrix cycles of a certain quasiquadratic system. Differential Equations, Vol. 33, 1997, p. 702-703.

[3] Andreev A. F., Andreeva I. A. Local study of a family of planar cubic systems. Vestnik St. Petersburg University: Mathematics, Seriya 1. Matematika, Mekhanika, Astronomiya, Vol. 2, 2007, p. 11-16.

[4] Andreev A. F., Andreeva I. A., Detchenya L. V., Makovetskaya T. V., Sadovskii A. P. Nilpotent centers of cubic systems. Differential Equations, Vol. 53, 2017, p. 1003-1008. 
[5] Andreev A. F., Andreeva I. A. Phase flows of one family of cubic systems in a Poincare circle I. Differential Equations and Control, Vol. 4, 2007, p. 17-26.

[6] Andreev A. F., Andreeva I. A. Phase flows of one family of cubic systems in a Poincare circle II. Differential Equations and Control, Vol. 1, 2008, p. 1-13.

[7] Andreev A. F., Andreeva I. A. Phase flows of one family of cubic systems in a Poincare circle III. Differential Equations and Control, Vol. 3, 2008, p. 39-54.

[8] Andreev A. F., Andreeva I. A. Phase flows of one family of cubic systems in a Poincare circle IV. Differential Equations and Control, Vol. 4, 2009, p. 181-213. 